\title{
The Value of Ensari's Proposal in Evaluating the Mucosal Pathology of Childhood Celiac Disease: Old Classification versus New Version
}

\author{
Servet Güreşci', Şamil Hızlı², Gülçin Güler Şimşek¹ \\ 'Department of Pathology, Keçiören Training and Research Hospital, Ankara, Turkey \\ ${ }^{2}$ Department of Pediatric Gastroenterology, Faculty of Medicine, Gaziantep University, Gaziantep, Turkey
}

\begin{abstract}
Objective: Small intestinal biopsy remains the gold standard in diagnosing celiac disease (CD); however, the wide spectrum of histopathological states and differential diagnosis of $C D$ is still a diagnostic problem for pathologists. Recently, Ensari reviewed the literature and proposed an update of the histopathological diagnosis and classification for CD.
\end{abstract}

Materials and Methods: In this study, the histopathological materials of 54 children in whom CD was diagnosed at our hospital were reviewed to compare the previous Marsh and Modified Marsh-Oberhuber classifications with this new proposal.

Results: In this study, we show that the Ensari classification is as accurate as the Marsh and Modified Marsh classifications in describing the consecutive states of mucosal damage seen in CD.

Conclusions: Ensari's classification is simple, practical and facilitative in diagnosing and subtyping of mucosal pathology of CD.

Key Words: Celiac disease, marsh classification, modified Marsh-Oberhuber classification, Ensari classification

Received: 06.12 .2011

Accepted: 23.02.2012

\section{Introduction}

Celiac disease (CD) is an autoimmune disorder of the small intestine triggered by the ingestion of gluten in genetically susceptible people. Despite new serological tests and genetic analysis, small intestinal biopsy remains the gold standard, especially for patients with mild mucosal abnormality (1). Rubin et al. (2) were the first investigators to classify CD histopathologically, although it was Marsh, a gastroenterologist, who clearly defined the pathophysiology of CD with histopathological classification (3). After that, Marsh-Oberhuber modified the classification scheme which is still widely used among pathologists $(4,5)$. Recent advances in clinical, genetic and therapeutic aspects of CD have made pathologists search for new histopathological diagnostic criteria and classifications. Recently, Ensari reviewed the literature and proposed an update to the Marsh classification from a pathologist's point of view (6). The aim of this study is to validate the accuracy and reproducibility of Ensari's proposal in the context of pediatric CD.

\section{Material and Methods}

In this retrospective study, the hospital records and histopathological archive materials of 54 children in whom CD was diagnosed at the departments of pathology and pediatric gastroenterology at Kecioren Training and Research Hospital between January 2007 and March 2010 were reviewed. The study protocol was approved by the Ankara Clinical Research Ethics Committee. The diagnosis was made according to the European Society for Pediatric Gastroenterology Hepatology and Nutrition (ESPGHAN) criteria, thus all biopsies were taken after antibody confirmation (7).

All endoscopies were performed by a single gastroenterologist who was aware that the patient had positive serology. Four endoscopic biopsies were taken from distal duodenum out of the bulbus. They were fixed in $10 \%$ formalin and embedded in paraffin. Serial sections ( $4 \mu \mathrm{m}$ thick) were obtained and stained with hematoxylin-eosin (H\&E). Slides were reevaluated according to the Marsh (MC) (3), modified MarshOberhuber (MMOC) (5), and proposed Ensari (EC) classification schemes (6) by two pathologists (SG and GGS), as shown in Table 1. The demographic characteristics, plasma tissue transglutaminase $\lg \mathrm{A}(\mathrm{tTG} \lg \mathrm{A}$ ) (reference range 0-10 $\mathrm{U} / \mathrm{mL}$ ) and anti-endomysial antibody IgA (EMA) (reference range $0-10 \mathrm{U} / \mathrm{mL}$ ) levels at the time of diagnosis were recorded.

\section{Data analysis}

Results were expressed as mean \pm standard deviation. The significance of intergroup differences was studied with SPSS (Statistical Package for the Social Sciences, version 15.0, 
SSPS Inc., Chicago, III, USA) by the Spearman rank correlation. A $p$ value $<0.05$ was considered statistically significant. Interobserver agreement was evaluated according to the concordance rate $(\%)$ and $\kappa$. For positive $\kappa$ values, the following interpretations are generally accepted: slight agreement, $\kappa=0.00-0.20$; fair agreement, $\kappa=0.21-0.40$; moderate agreement, $\kappa=0.41-0.60$; substantial agreement, $\kappa=0.61-0.80$; and almost perfect agreement, $\kappa=0.81-1.00$ (8).

\section{Results}

Forty of the 54 patients were female (74\%). The mean age at the time of diagnosis was $8.9 \pm 4.39$. Mean plasma tTG $\operatorname{lgA}$ and EMA levels were $163.12 \pm 72.4 \mathrm{IU} / \mathrm{mL}$ and $140.29 \pm 67.5 \mathrm{IU} /$ $\mathrm{mL}$, respectively.

As a result of the histopathological examination, with regard to $\mathrm{MC}$, two cases (3.7\%) were type 1 and 52 cases (96.2\%) were type 3 . There were no type 2 cases. With regard to MMOC, two cases $(3.7 \%)$ were type 1 , no cases were type 2 , two cases (3.7\%) were type $3 \mathrm{~A}, 15$ cases $(27.7 \%)$ were type $3 \mathrm{~B}$ and 35 cases were $(64.8 \%)$ type $3 \mathrm{C}$. By EC, there were two (3.7\%) type 1, 17 (31.5\%) type 2 and 35 (64.8\%) type 3 patients.

Plasma tissue transglutaminase IgA levels showed a significant correlation with MMOC $(p=0.002)$ and $E C(p<0.001)$, but the correlation with $\mathrm{MC}$ was not significant $(p>0.05)$. EMA levels revealed a weak correlation only with EC ( $p=0.048)$, but a correlation with the other classification schemes was not achieved.

Ensari's proposal demonstrated a strong correlation with both MC $(p<0.001)$ and MMOC $(p=0.003)$. The correlations between EC and the other valuables are shown in Table 2.

In the assessment of interobserver agreement, $\kappa$ was 1 (perfect agreement) and the concordance rate was $100 \%$ among two pathologists for $\mathrm{MC}$ and $\mathrm{EC}$. With regard to MMOC, $\kappa$ was 0.558 (moderate agreement) and the concordance rate was $79.6 \%$.

\section{Discussion}

In this study, we evaluated the histopathological features of 54 pediatric patients with $C D$ in the context of three classifi- cation schemes: MC, MMOC and EC. In our study, the disease was found to be more frequent in females with a female-tomale ratio of 7:20. The most common age of diagnosis was 8-9 years, which is school age. This result is consistent with previous studies performed in Turkey (9-11).

It should be questioned whether classifying the histopathological states of CD is of any prognostic or clinical use, but in daily practice, pathologists are almost always asked to stage the mucosal pathology of $C D$, both for diagnosis and to assess regression after adopting a gluten-free diet (1). However, the wide spectrum of histopathological states and differential diagnosis of CD is still a diagnostic problem for pathologists.

The Marsh classification defines type 1 (infiltrative lesion) as a normal mucosa with increased intraepithelial lymphocyte (IEL) count, type 2 (hyperplastic lesion) as crypt hyperplasia with normal villi with a raised IEL count, type 3 (destructive lesion) as flat mucosa with crypt hyperplasia and a raised IEL count and type 4 (atrophic lesion) as flat mucosa with crypt hypoplasia and mild inflammation (3). This classification is based on architectural changes in the mucosa achieved under experimental conditions and does not define a cutoff number for $\operatorname{IELs}(4,6)$. On the other hand, MMOC, which is the modified version of MC proposed seven years after it was first presented, kept type 1 and type 2 constant, but subcategorized destructive lesions (type 3 ) into three additional subgroups: mild, moderate and severe villous atrophy, without defining the exact ratio of villous shortening $(5,6,12)$. This classification is widely used among pathologists today. However, it is known that increasing the number of diagnostic categories leads to lower interobserver and intraobserver agreement with a consequential reduction in diagnostic

Table 2. Correlation coefficients between Ensari's proposal and other variables. (Spearman rank correlation test)

\begin{tabular}{|lll|}
\hline Measurements & $\mathbf{r}$ & $\mathbf{p}$ \\
\hline $\mathrm{MC}$ & 0.993 & $<0.001$ \\
\hline $\mathrm{MMOC}$ & 0.392 & 0.003 \\
\hline $\mathrm{tTG} \operatorname{lgA}(\mathrm{IU} / \mathrm{mL})$ & 0.459 & $<0.001$ \\
\hline EMA $(\mathrm{IU} / \mathrm{mL})$ & 0.281 & 0.048 \\
\hline
\end{tabular}

Table 1. Comparison of classification systems used for diagnosing celiac disease, (IELC: intraepithelial lymphocyte count)

\begin{tabular}{|c|c|c|}
\hline *Marsh Classification (1992) & $\begin{array}{l}\star \star \text { Modified Marsh-Oberhuber } \\
\text { Classification (1999) }\end{array}$ & 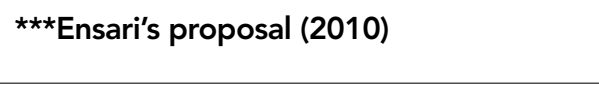 \\
\hline Type 1 (normal villi+IELC $\uparrow$ ) & Type 1 (normal villi+IELC $\uparrow$ ) & Type 1 (normal villi+IELC $\uparrow$ ) \\
\hline $\begin{array}{l}\text { Type } 2 \text { (normal villi+ } \\
\text { crypt hyperplasia+IELC } \uparrow \text { ) }\end{array}$ & $\begin{array}{l}\text { Type } 2 \text { (normal villi+ } \\
\text { crypt hyperplasia+IELC } \uparrow \text { ) }\end{array}$ & Type 1 \\
\hline \multirow[t]{3}{*}{$\begin{array}{l}\text { Type } 3 \text { (flat mucosa+ } \\
\text { crypt hyperplasia+IELC } \uparrow \text { ) }\end{array}$} & $\begin{array}{l}\text { Type 3A (mild villous shortening } \\
\text { +crypt hyperplasia+IELC } \uparrow \text { ) }\end{array}$ & $\begin{array}{l}\text { Type } 2 \text { (short villi+crypt hyperplasia+IELC } \uparrow \text { ) } \\
\text { crypt hyperplasia+ IELC } \uparrow \text { ) }\end{array}$ \\
\hline & $\begin{array}{l}\text { Type 3B (moderate villous shortening + } \\
\text { crypt hyperplasia+IELC } \uparrow \text { ) }\end{array}$ & Type 2C \\
\hline & $\begin{array}{l}\text { Type } 3 C \text { (severe villous shortening + } \\
\text { crypt hyperplasia+IELC } \uparrow \text { ) }\end{array}$ & $\begin{array}{l}\text { Type } 3 \text { (flat mucosa+ crypt } \\
\text { hyperplasia+ IELC } \uparrow \text { ) }\end{array}$ \\
\hline Type 4 (atrophic mucosa) & Type 4 (atrophic mucosa) & obsolete \\
\hline
\end{tabular}




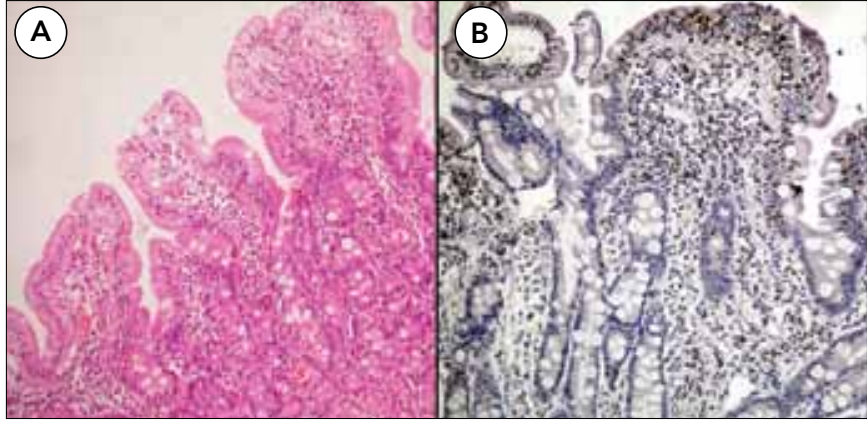

Figure 1. Intraepithelial lymphocytosis, with crypt hyperplasia and mild villous shortening. Type 3 for MC, type $3 A$ for MMOC and type 2 for EC

(A: HE 200x, B: anti CD3 immunostaining- streptavidin-biotinperoxidase 200x)

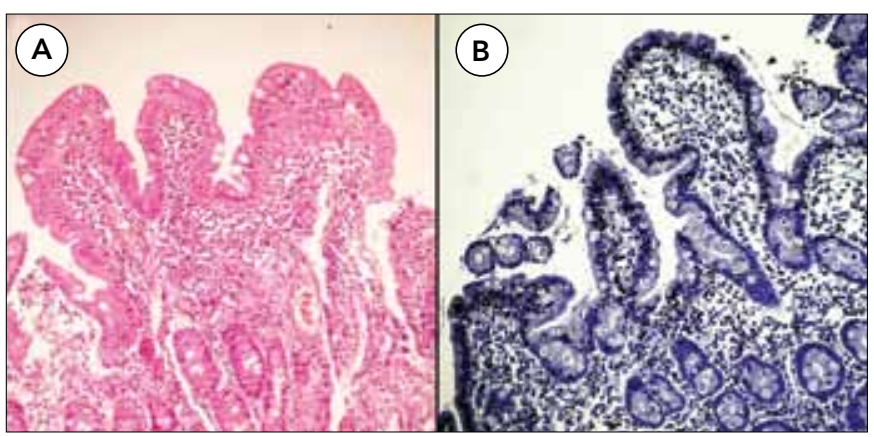

Figure 2. Intraepithelial lymphocytosis with crypt hyperplasia and marked villous shortening. Type 3 for MC, type 3B for MMOC and type 2 for EC

(A: HE 200x, B: anti CD3 immunostaining- streptavidin-biotin-peroxidase 200x)

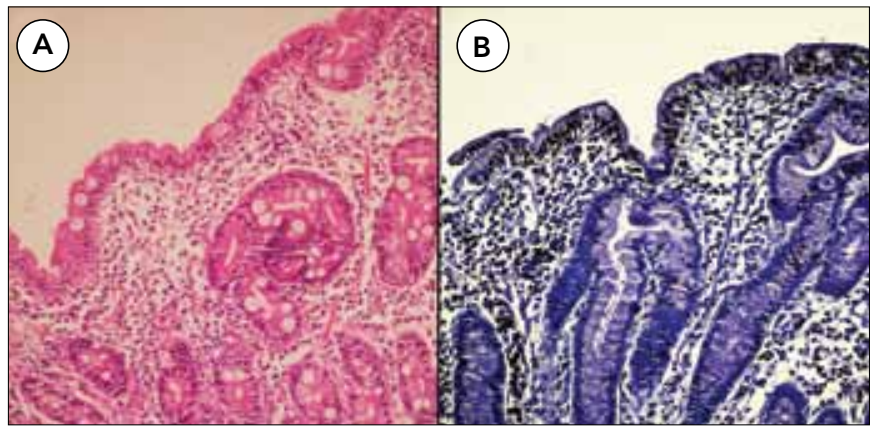

Figure 3. Diffuse intraepithelial lymphocytosis with crypt hyperplasia and total villous shortening. Type 3 for both MC and EC, but type 3C for MMOC

(A: HE 200x, B: anti CD3 immunostaining- streptavidin-biotin-peroxidase 200x)

reproducibility (12). Also, poorly oriented specimens may result in a failure to highlight minor differences in villous height, which may be problematic in distinguishing type $3 \mathrm{~A}$ and $3 \mathrm{~B}$ lesions. Although the overall classification is based on the highest type that is observed, heterogeneity in the morphology of mucosal pathology is another factor that contributes to interobserver variability (4). These make MMOC somewhat subjective and unreliable $(12,14)$. Moreover, MMOC defines a cutoff IEL number of 40 per 100 enterocytes derived from jejunal biopsies
(5), which is very high for more recently defined duodenal IEL numbers (20 per 100 enterocytes in H\&E sections and 25 per 100 enterocytes in immunohistochemical sections) (15-17). Setting a higher cutoff point for IEL number can lead to underdiagnosing Marsh type 1 lesions, which is sometimes negative for serology, but is always normal architecturally $(6,12,18)$. It is important to recognize CD patients with mild morphologic changes because they can develop iron deficiency anemia and osteoporosis (19). An incorrect diagnosis of CD is not only a risk for the patient or healthy subject, but also a waste of resources, time and money for institutions (18). Recently, Ensari updated the $\mathrm{MC}$ with respect to these major pitfalls (6).

Ensari's proposal comprises three groups, and designates mucosal pathology as normal villi with IELosis (type 1), shortened villi with IELosis and crypt hyperplasia (type 2) and completely flat mucosa with IELosis and crypt hyperplasia (type 3) (6). The scheme does not define the type 4 lesion used in $\mathrm{MC}$ as it has been proven to be a neoplastic disease by molecular techniques $(6,12)$. Ensari emphasized that $M C$ type 2 lesions are mainly observed under strict experimental conditions and, in practice, crypt hyperplasia is always accompanied by villous shortening, such that type 2 for MC/MMOC should no longer be used in routine practice. Although our sample number is relatively low, we did not diagnose any type 2 lesions according to $\mathrm{MC}$ or MMOC, which is consistent with Ensari's statement. EC, by not having the subtypes defined in MMOC type 3, facilitated the histopathological diagnosing process and reduced interobserver variability in our study. This was shown by the perfect agreement among the two observers using EC, whereas MMOC showed only moderate agreement. Variability was especially higher, as expected, in type $3 \mathrm{~A}$ and $3 \mathrm{~B}$ lesions in MMOC which are both designated as type 2 in EC (Figure 1-3).

Elevated IEL counts have been observed in $1.3 \%$ to $2.2 \%$ of patients undergoing upper gastrointestinal endoscopy and small intestinal biopsy (20). Since it can be seen in various pathological processes, like giardiasis and Helicobacter pyloriassociated duodenitis which are frequent in Turkey, IELosis is not a diagnostic but rather a suggestive feature of $C D$. In the normal mucosa IELs, are distributed along the villi in a decreasing pattern from the base of the villus toward the villous tip (21). An alternative method which has been proposed for detecting IELs recommends counting IELs in the villous tips, such that 6-12 IELs per 20 enterocytes is considered suggestive of $C D$ (22). Although this method clearly simplifies the examination, Ensari recommends that the distribution pattern of IELs is more valuable than the exact counts. Especially diffuse infiltration of the villous epithelium by lymphocytes is a characteristic feature of $C D$ (6). As a result of studies to find the best method of counting IELs, routine application of CD3 immunohistochemistry has been proposed when there a normal mucosa is present (15). Ensari emphasizes that immunohistochemistry should be used when there is a suspected rather than definite increase in IELs (6). In our study, we benefited from the experience of Ensari, so the examination process was very fast and easy for us.

In this study, by obtaining a correlation with both $\mathrm{MC}$ and MMOC, we showed that EC is as accurate as these two classification schemes in the consecutive states of mucosal damage 
seen in CD. Furthermore, neither $\mathrm{MC}$ nor MMOC but $\mathrm{EC}$ revealed a correlation with both tTG IgA and EMA levels, which are specific and sensitive antibodies used to diagnose $C D$. This means that when mucosal pathology becomes worse, antibody levels increase. Therefore, the clinician can make a suggestion about the mucosal pathology according to these antibody levels during follow-up with the patient. Thus, the EC scheme is more valuable from a clinical point of view.

\section{Conclusion}

EC is simple, practical and facilitative in the diagnosis and subtyping of the mucosal pathology of $C D(6)$. We believe that this classification scheme will simplify the internationally-used terminology, improve interobserver and intraobserver variability and facilitate the relationship between pathologists and clinicians. The value of EC will be better understood after being used in a wider series.

\section{Conflict of Interest}

No conflict of interest was declared by the authors.

\section{References}

1. Brown I, Mino-Kenudson M, Deshpande V, Lauwers GY. Intraepithelial lymphocytosis in architecturally preserved proximal small intestinal mucosa: an increasing diagnostic problem with a wide differential diagnosis. Arch Pathol Lab Med 2006;130:1020-5.

2. Rubin CE, Brandborg LL, Phelps PC, Taylor HC Jr. Studies of celiac disease.l. The apparent identical and specific nature of the duodenal proximal jejunal lesion in celiac disesase and idiopathic sprue. Gastroenterology 1960;38:28-49.

3. Marsh MN. Gluten, major histocompatibility complex, and small intestine. A molecular and immunobiologic approach to the spectrum of gluten sensitivity ('celiac sprue'). Gastroenterology 1992;102:330-5

4. Dickson BC, Streutker CJ, Chetty R. Coeliac disease: an update for pathologists. J Clin Pathol 2006;59:1008-16. [CrossRef]

5. Oberhuber G, Granditsch G, Vogelsang H. The histopathology of celiac disease: time for a standardized report scheme for pathologists. Eur J Gastroenterol Hepatol 1999;11:1185-94. [CrossRef]

6. Ensari A. Gluten-sensitive enteropathy (celiac disease): controversies in diagnosis and classification. Arch Pathol Lab Med 2010;134:826-36.
7. Revised criteria for diagnosis of coeliac disease. Report of Working Group of European Society of Pediatric Gastroenterology and Nutrition. Arch Dis Child 1990; 65: 909-11. [CrossRef]

8. Wells WA, Carney PA, Eliassen MS, Grove MR, Tosteson AN. Pathologists' agreement with experts and reproducibility of breast ductal carcinoma-in-situ classification schemes. Am J Surg Pathol 2000;24:651-9. [CrossRef]

9. Balamtekin N, Uslu N, Baysoy G, Usta Y, Demir H, Saltik-Temizel IN, et al. The presentation of celiac disease in 220 Turkish children. Turk J Pediatr 2010;52:239-44.

10. Kuloglu Z, Kirsaclioglu CT, Kansu A, Ensari A, Girgin N. Celiac disease: presentation of 109 children. Yonsei Med J 2009;50:617-23. [CrossRef]

11. Ertekin V, Selimoglu MA, Kardas F, Aktas E. Prevalence of celiac disease in Turkish children. J Clin Gastroenterol 2005;39:689-91. [CrossRef]

12. Corazza GR, Villanaci V. Coeliac disease. J Clin Pathol 2005;58:573-4. [CrossRef]

13. Weile B, Hansen BF, Hagerstrand I, Hansen JP, Krasilnikoff PA. Interobserver variation in diagnosing coeliac disease. A Joint study by Danish and Swedish pathologists. APMIS 2000;108:380-4. [CrossRef]

14. Di Sabatino A, Corazza GR. Coeliac disease. Lancet 2009;373:1480-93. [CrossRef]

15. Veress B, Franzen L, Bodin L, Borch K. Duodenal intraepithelial lymphocyte-count revisited. Scand J Gastroenterol 2004;39:138-44. [CrossRef]

16. Hayat M, Cairns A, Dixon MF, O'Mahony S. Quantitaion of intraepithelial lymphocytes in human duedonum: what is normal? J Clin Pathol 2002;55:393-4. [CrossRef]

17. Pellegrino S, Villanacci V, Sansotta N, Scarfi R, Bassotti G, Vieni G, et al. Redefining the intraepithelial lymphocytes threshold to diagnose gluten sensitivity in patients with architecturally normal duodenal histology. Aliment Pharmacol Ther 2011;33:697-706. [CrossRef]

18. Biagi F, Bianchi PI, Campanella J, Zanelatti G, Corazza GR. The impact of misdiagnosing celiac disease at a referral centre. Can J Gastroenterol 2009;23:543-5.

19. Brown I, Kenudson MM, Deshpande V, Lauwers GY. Intraepithelial lymphocytosis in architecturally preserved proximal small intestinal mucosa: an increasing diagnostic problem with a wide differential diagnosis. Arch Pathol Lab Med 2006;130:1020-5.

20. Mahadeva S, Wyatt JI, Howdle PD. Is a raised intraepithelial lymphocyte count with normal duodenal villus architecture clinically relevant? J Clin Pathol 2002;55:424-8. [CrossRef]

21. Goldstein NS. Proximal small bowel mucosal villous intraepithelial lymphocytes. Histopathology 2004;44:199-205. [CrossRef]

22. Biagi F, Luinetti O, Campanella J, Klersy C, Zambelli C, Villanacci V, et al. Intraepithelial lymphocytes in the villous tip: do they indicate potential celiac disease? J Clin Pathol 2004;57:835-9. [CrossRef] 